\title{
The Effects of prewarmed endotracheal tubes and 2\% lidocaine jelly on postoperative sore throat
}

\author{
Song Jia M.D. ${ }^{1}$ Jang Eun-a M.D. ${ }^{1}$ \\ Department of Anesthesiology and Pain Medicine, Chonnam National University Hospital, Gwangju, South \\ Korea
}

Sore throat is one of the most common complications after tracheal intubation. And it is a common reason for patients to avoid undergoing subsequent general anaesthesia, and it adversely affects patient satisfaction and activity after leaving the hospital. Hence, we conducted a prospective, randomised trial in patients undergoing laparoscopic surgery (laparoscopic cholecystectomy or laparoscopic cystectomy) to evaluate the effects of the combination of thermally softened tubes and lidocaine jelly on the incidence and severity of POST.

Through prospective, double-blind, single-centre, randomised controlled trial, in total, our study included 144 patients aged 2070 years who were classified as American Society of Anesthesiologists physical status I-III scheduled for laparoscopic surgery as an elective procedure. Based on our exclusion criteria, we removed patients with: 1) anticipated difficult intubation (Mallampati class $\geq 3$ ), 2) previous throat pain or hoarseness history, 3) symptoms of an upper airway infection, 4) habit of smoking, 5) cervical spine disease and 6) a history of lidocaine allergy. Patients who required repetitive attempts or stylets or Backwards Upwards Rightwards Pressure (BURP) manoeuvres at intubation were also excluded from the analysis. The study was conducted by two sub-investigators and one assistant in a blinded manner. The assistant, who was unaware of the protocol, prepared each endotracheal tube (ETT). The first investigator performed intubation, and the second investigator collected the variables. Computer-generated codes were used for random group assignment:

Group A, room temperature ETT with saline;

Group B, room temperature ETT with lidocaine jelly;

Group C, thermally softened ETT with saline

Group D, thermally softened ETT with lidocaine jelly.
ETT used in Groups $C$ and D was softened by warming at $40^{\circ} \mathrm{C}$. Sterile TaperGuard oral tracheal tubes (Covidien, $8.0 \mathrm{~mm}$ internal diameter for male patients and $7.0 \mathrm{~mm}$ internal diameter for female patients) were used with Macintosh \#3 laryngoscopes. The intracuff pressure was measured immediately after intubation at 20 to $25 \mathrm{cmH} 2 \mathrm{O}$ using a manometer. At the end of surgery, tracheal and oral suction was performed gently before extubation. Immediately and $1 \mathrm{~h}$ after arrival at the recovery room and $6 \mathrm{~h}, 24 \mathrm{~h}$ and $48 \mathrm{~h}$ after tracheal extubation, the second investigator collected the incidence of POST. The degree of sore throat severity was measured using a visual analogue scale (VAS).

The sample size calculation for the present study was based on a pilot study $(n=32)$. All statistical analyses were performed with SPSS.

Table 2. Severity of postoperative sore throat

\begin{tabular}{llllll}
\hline & Group A & Group B & Group C & Group D & P value \\
\hline At 0h & $2.4 \pm 1.0$ & $2.4 \pm 1.0$ & $2.2 \pm 0.7$ & $2.9 \pm 1.3$ & 0.14 \\
At 1h & $1.9 \pm 0.8$ & $1.7 \pm 0.6$ & $1.9 \pm 0.6$ & $2.1 \pm 1.6$ & 0.73 \\
At 6h & $1.7 \pm 0.9$ & $2.7 \pm 1.5$ & $1.7 \pm 0.6$ & $2.9 \pm 1.6$ & 0.19 \\
At 24h & $2.0 \pm 1.4$ & 2.0 & $1.0 \pm 0.0$ & 2.0 & 0.80 \\
\hline
\end{tabular}

The overall incidence of POST was significantly lower with the combination of thermally softened tubes and lidocaine jelly compared to either intervention alone. However, there were no differences in the severity of sore throat or incidence of hoarseness and other adverse effects

\begin{tabular}{|c|c|c|c|c|c|c|}
\hline & $\begin{array}{l}\text { Group A } \\
(\mathrm{n}=34)\end{array}$ & $\begin{array}{l}\text { Group B } \\
(\mathrm{n}=33)\end{array}$ & $\begin{array}{l}\text { Group C } \\
(\mathrm{n}=35)\end{array}$ & $\begin{array}{l}\text { Group D } \\
(\mathrm{n}=35)\end{array}$ & $P$ value & $\begin{array}{l}\text { Table } 1 . \\
\text { Incidences of postoperative sore throat, } \\
\text { hoarseness }\end{array}$ \\
\hline \multicolumn{7}{|c|}{ Sore throat } \\
\hline Oh & $26(76 \%)^{*}$ & $26(79 \%)^{*}$ & $25(71 \%)$ & $17(49 \%)$ & 0.027 & \\
\hline $1 \mathrm{~h}$ & $20(59 \%)$ & $19(58 \%)$ & $22(63 \%)$ & $18(51 \%)$ & 0.810 & \\
\hline $24 h$ & $4(12 \%)$ & $3(9 \%)$ & $4(11 \%)$ & $2(6 \%)$ & 0.855 & \\
\hline $48 \mathrm{~h}$ & $0(0 \%)$ & $0(0 \%)$ & $1(3 \%)$ & $0(0 \%)$ & 0.402 & \\
\hline \multicolumn{6}{|c|}{ Hoarseness } & $\begin{array}{l}\text { Values are number of patients (percentage). } \\
{ }^{*} \mathrm{P}<0.05 \text { compared with Group } D .\end{array}$ \\
\hline Overall & 19 (55.9\%) & $15(45.5 \%)$ & $15(42.9 \%)$ & $16(45.7 \%)$ & 0.715 & \\
\hline
\end{tabular}

